\title{
Meeting Report: Controlled and Modified Drug Release
}

\author{
Vivian A. Gray \\ Dissolution Technologies, Inc., Hockessin, DE
}

e-mail:vagray@rcn.com

T he Inaugural Controlled and Modified Drug Release Conference took place in Philadelphia, PA, on May 7 and 8,2014 . This conference provided case studies and take-home examples of the latest technologies and methods for improving drug release mechanisms. Additionally, the workshop provided the latest regulatory guidance update and the implications for industry. This conference had excellent audience participation and a variety of speakers and topics. The program is listed below.
There were also two preconference workshops on May 7, 2014. Duk Soon Choi, Ph.D., and Navnit Shah, Ph.D., both of Kashiv Pharma, presented Workshop A. The topic was Imaging and Characterization Techniques for Drug Delivery: Systems and Targeted Drug Delivery. Jim Jingjun Huang, Ph.D., of Ascendia Pharmaceutical, presented Workshop B. The topic was Rational Design of Drug Delivery Systems for Poorly Water Soluble Drugs.

The second conference on Controlled and Modified Drug Release will be held on May 6-7, 2015, in Philadelphia, PA. For more information, visit www.curtiscoulter.com.

\begin{tabular}{|c|c|c|}
\hline Date & Speaker & Topic \\
\hline \multirow[t]{7}{*}{7 May 2014} & $\begin{array}{l}\text { Steven Sutton, Ph.D. } \\
\text { University of New England }\end{array}$ & $\begin{array}{l}\text { Opening Discussion: Regulatory Challenges and Opportunities for the Future of Controlled \& } \\
\text { Modified Release }\end{array}$ \\
\hline & $\begin{array}{l}\text { Sherry Ku, Ph.D. } \\
\text { TWi Biotech \& TWi Pharmaceuticals }\end{array}$ & Understanding and Establishing the Right Considerations for IVIVC Correlations \\
\hline & $\begin{array}{l}\text { Mengwei Hu, Ph.D. } \\
\text { Merck \& Co. }\end{array}$ & $\begin{array}{l}\text { Pre-investment in Controlled Release: Discovery Controlled Release: Assessment and } \\
\text { Formulation Strategies }\end{array}$ \\
\hline & $\begin{array}{l}\text { Jon Mole } \\
\text { Sirius Analytical, Inc. }\end{array}$ & Automated Measurements of Supersaturation, Biphasic Dissolution and Lipolysis \\
\hline & $\begin{array}{l}\text { Ravi Shanker, Ph.D. } \\
\text { Pfizer, Inc. }\end{array}$ & Improving Oral Bioavailability for Poorly Water-soluble Drug Candidates \\
\hline & $\begin{array}{l}\text { Able A.Creasey, Ph.D. } \\
\text { Janssen Research \& Development }\end{array}$ & $\begin{array}{l}\text { Successful Delivery of Small and Large Molecules using Polymers and in Localized/Sustained } \\
\text { Release Manner }\end{array}$ \\
\hline & $\begin{array}{l}\text { Vikas Agarwai, Ph.D. } \\
\text { Taris Biomedical }\end{array}$ & Controlled Local Delivery of Drugs to the Urinary Bladder \\
\hline \multirow[t]{10}{*}{8 May 2014} & $\begin{array}{l}\text { Steven C. Sutton, Ph.D. } \\
\text { University of New England }\end{array}$ & Predictive Animal Models for Assessing Long-Acting Formulations for Human and Animal Health \\
\hline & $\begin{array}{l}\text { Michael J.Valazza, R.Ph. } \\
\text { Catalent Pharma Solutions }\end{array}$ & Advanced Technologies for Enabling Better Treatments through Oral Drug Delivery \\
\hline & $\begin{array}{l}\text { Weijia Zheng, Ph.D. } \\
\text { AstraZeneca }\end{array}$ & Application of Controlled release Approaches in Preclinical Stage \\
\hline & $\begin{array}{l}\text { James DiNunzio, Ph.D. } \\
\text { Merck \& Co. }\end{array}$ & New Formulations Hot-Melt Extrusion Technology Development to Optimize Sustained Delivery \\
\hline & $\begin{array}{l}\text { Daniel Schweizer, Ph.D. } \\
\text { Novartis AG }\end{array}$ & Sustained Delivery of Therapeutic Antibodies \\
\hline & $\begin{array}{l}\text { Der-Yang Lee, Ph.D. } \\
\text { Johnson \& Johnson Consumer \& Personal } \\
\text { Products }\end{array}$ & Drug Delivery Applications of Ion Exchange Resins \\
\hline & $\begin{array}{l}\text { Michelle Howard-Sparks, Ph.D. } \\
\text { Zafgen, Inc. }\end{array}$ & $\begin{array}{l}\text { Examining the Use of Nanoparticles for Modified release and Targeted Delivery for Compounds } \\
\text { of Poor Solubility and Permeability }\end{array}$ \\
\hline & $\begin{array}{l}\text { Jin Zhang, Ph.D. } \\
\text { Novartis Pharmaceuticals Corporation }\end{array}$ & $\begin{array}{l}\text { Combining In-Vivo Animal Models with Physiologically Based Pharmacokinetics (PBPK) } \\
\text { Modeling to Effectively Develop Modified Release Dosage Forms }\end{array}$ \\
\hline & $\begin{array}{l}\text { Vrushali Waknis, Ph.D. } \\
\text { Bristol-Myers Squibb } \\
\text { S. Kamal Jonnalagadda, Ph.D. } \\
\text { Philadelphia College of Pharmacy, } \\
\text { University of the Sciences }\end{array}$ & Controlled Drug Release using Biodegradable Polymers \\
\hline & $\begin{array}{l}\text { Jeffrey Liu, Ph.D. } \\
\text { GlaxoSmithKline }\end{array}$ & $\begin{array}{l}\text { Developing New Sustained-Release Formulations via Exploiting In-Vivo Modeling \& Simulations } \\
\text { (IVMS) }\end{array}$ \\
\hline
\end{tabular}

